\title{
Presupposition projection from disjunction is symmetric
}

\author{
Alexandros Kalomoiros \& Florian Schwarz*
}

\begin{abstract}
The role of linear order for presupposition projection is a key theoretical question, but the empirical status of (a-)symmetries in projection from various connectives remains controversial. We present experimental evidence that presupposition projection from disjunction is symmetric. 'Bathroom disjunctions', where either disjunct seems able to support a presupposition in the other if its negation entails it, have been argued to be evidence for symmetric projection; but there are alternative theoretical options. Adapting the paradigm of Mandelkern et al. (2020) for projection from conjunction, our experimental data supports the view that we are dealing with genuinely symmetric projection from disjunction. This contrasts with Mandelkern et al.'s findings for asymmetric projection from conjunction, and thus provides evidence for variation in projection (a-)symmetry across connectives, contra accounts proposing general accounts predicting uniform asymmetry effects due to left-to-right processing (e.g., Schlenker 2009).
\end{abstract}

Keywords. disjunction; presupposition projection; processing asymmetries

1. Introduction. In this paper we present experimental results aimed at testing the projection behavior of presupposition triggers in Partee's so-called 'bathroom disjunctions', as in (1):

(1) a. Either the bathroom is in a weird place or this house has no bathroom.

b. Either this house has no bathroom or the bathroom is in a weird place.

The definite the bathroom presupposes that the house has a bathroom, but the whole sentence does not carry such a presupposition, apparently because the other disjunct explicitly raises the possibility of there not being one. On one view, such examples have been taken to show that disjunctions exhibit symmetric presupposition filtering, i.e., that both preceding and following material in a complex sentence is considered in evaluating the presupposition; here, the negation of the other disjunct is what is considered in assessing the presupposition, and as it entails the existence of a bathroom, the presupposition of the definite is supported sentence internally, and thus 'filtered' (in Karttunen's terminology) and not present as a presupposition of the complex sentence as a whole. Positing the availability of both 'left-to-right' and 'right-to-left' filtering in bathroom disjunctions then constitutes a challenge for accounts of presupposition projection which claim that the filtering mechanism is fundamentally asymmetric (i.e. it proceeds from left-to-right and not from right-to-left, at least by default).

However, alternative explanations of the pattern in (1) have been proposed to rescue such accounts. There are two main candidate approaches to bathroom disjunctions in the literature that argue for some level of asymmetry based on linear order in (1). We'll consider these approaches in the specific form proposed in two directly relevant papers: Schlenker (2009) proposes that filtering is asymmetric by default, due to a processing preference for left-to-right filtering, but that symmetric filtering is in principle available though it incurs a processing cost.

\footnotetext{
* Thanks to the members of the Penn Semantics lab and to the participants of the Fall 2018 seminar on presupposition projection at Penn for useful comments at various stages of this project. All errors are our own. Authors: Alexandros Kalomoiros, University of Pennsylvania (akalom@sas.upenn.edu) \& Florian Schwarz, University of Pennsylvania (florians@sas.upenn.edu).
} 
In contrast, Hirsch \& Hackl (2014) argue that in fact, the presupposition projection mechanism involves genuinely asymmetric projection from disjunction across the board, and explains the apparent symmetry in bathroom disjunction by appealing to local accommodation in apparent cases of right-to-left filtering, which is resorted to in order to satisfy general pragmatic constraints associated with disjunction, and which provides an alternative mechanism to prevent the presupposition from being globally present for the complex sentence as a whole.

Our experiment aims to test the more fine-grained differences in predictions of the two main analytical options - symmetric vs. (at least by default) asymmetric projection from disjunction. We adapt and extend the methodological approach in Mandelkern et al. (2020), who found a strong asymmetry in the projection behavior of conjunction. We carry over their paradigm to the domain of disjunction. Our results indicate that bathroom disjunctions indeed constitute cases of genuinely symmetric projection from disjunction: it makes no difference in terms of acceptability judgments if the presupposition is in the first or the second disjunct, in contrast to Mandelkern et al.'s findings for conjunction. We conclude that these differences support an approach that ties the projection behavior of each connective to its lexical properties, rather than an approach that posits a domain-general projection mechanism that predicts uniform order effects across connectives, with asymmetry of one sort or another at play in bathroom sentences, and corresponding predictions on acceptability judgments.

The rest of this paper is organized as follows: Sections 2 provides the relevant theoretical and experimental background, introducing the basic notions of presupposition, projection and filtering. In particular, we review Schlenker (2009)'s account of local contexts, and its predictions for disjunction. We contrast this with the alternative view as laid out by Hirsch \& Hackl (2014) . Finally, we review the prior experimental results for conjunction in Mandelkern et al. (2020). Sections 3 presents our new experiment on disjunction, and the theoretical implications of our findings for theories of presupposition projection. Section 4 concludes.

\section{Background.}

\subsection{Presupposition AND Filtering.}

2.1.1. BASIC Notions. Certain lexical item are associated with presuppositions, and accordingly seem to generally require certain information to be established in the utterance context for their use to be felicitous. For example, the verb continue is generally taken to presuppose that whatever state (or activity) is being described as continuing was indeed something that held (or was going on) before the referenced time:

(2) \# John continues having research interests in Tolkien.

Uttering (2) out of the blue, in a conversation where it does not count as established that John has had prior research interests in Tolkien, seems to give rise to some amount of infelicity. Now contrast (2) with (3) below:

(3) I saw my old friend from college John the other day. We had done a research project on Tolkien together back then. To my surprise, he continues to have research interests in Tolkien.

In (3), the information that John had prior research interests in Tolkien is introduced explicitly before the sentence with continue, ensuring that the discourse supports the presupposition and is therefore fully felicitous. We say that 'continues having research interests' presupposes 'hav- 
ing had prior research interests'; the main new information that it contributes to an assertion is that of 'having current research interests'. The infelicitous (2), where the presupposition of 'continue' is not part of the context, is then a case of 'presupposition failure.'. In contrast, in (3), the presupposition is explicitly supported by the global discousre context.

A key challenge in the literature on presupposition projection concerns the behavior of presuppositions in embedded contexts, e.g., in the scope of logical connectives. In some cases, the presuppositions seem to become presuppositions of the complex sentence as a whole, whereas in others, they don't - this is the 'projection problem' for presuppositions. Consider the following contrast in conjunctions:

(4) a. \#John continues having research interests in Tolkien and he had prior research interests in Tolkien.

b. John had prior research interests in Tolkien and he continues having research interests in Tolkien.

When the first conjunct introduces the presupposition, (4-b), the sentence as a whole seems felicitous, even in absence of a supporting discourse context, in contrast to (2), suggesting that the sentence as a whole does not carry the presupposition introduced by continue. However, when the first conjunct contains the trigger and the second conjunct introduces the information supporting the presupposition, then infelicity ensues, (4-a). ${ }^{1}$

Intuitively, the presupposition trigger in (3) is felicitous because the presupposition is already entailed by the larger context in which the discussion is being conducted, i.e., it is part of the common ground as the trigger is evaluated (Stalnaker (1974)). This intuition can easily be extended to the data in (4), as suggested by Stalnaker: As a hearer hears (4-b), they first parse the first conjunct followed by 'and'. At this point, they can already add the information that John had prior research interests in Tolkien to the global context represented by the common ground (i.e. the set of worlds compatible with what is commonly assumed by the discourse participants.). As they go on to parse the second conjunct, they thus can evaluate its presupposition relative to an updated global context that integrates the information of the first conjunct. That context entails that John had prior research interests in Tolkien, meaning the presupposition is supported and its use felicitous. Since the support in this case is introduced sentence-internally, the overall conjunctive sentence comes with no relevant constraints on the contexts it can be used in - the presupposition in the second conjunct gets filtered, in the terminology of Karttunen (1973). In contrast, in (4-a), the first conjunct gets parsed against the global context. In order to be felicitous, the presupposition about John's prior research interests has to be entailed by that context as it gets evaluated. In other words, it projects, i.e., the conjunction as a whole carries the same presupposition as the simple sentence in (2). The second conjunct, which contains the presupposition, seems to 'come too late' to make a difference. Hence infelicity arises in absence of global contextual support of the presupposition.

Note that on this general view on the projection problem, the context relative to which a presupposition in a complex sentence is evaluated can include information introduced by other parts of the same overall sentence. This is the 'local context' (Karttunen 1973; and much subsequent work). The question of how to precisely and systematically define what counts as the

\footnotetext{
${ }^{1}$ The empirical picture may be more nuanced here due to other factors at play, but we will not get into this here; see Mandelkern et al. (2020), which we also discuss below, for detailed discussion and experimental data addressing potential issues and confounds.
} 
local context in a given embedded environment is at the heart of theoretical accounts of presupposition projection, and we'll turn to some detailed proposals shortly. However, taking an intuitive characterisation of 'local context' as have sketched above for granted for the moment, the key generalization about presupposition projection can be stated as follows:

A presupposition projects iff it is not entailed by its local context.

In (4-a), the local context is simply the global context, so the constraints the presupposition trigger places on its local context are automatically constraints on the global context as well. However, in (4-b) the local context is the initial global context plus the information contained in the first conjunct. Since in the case at hand, the first conjunct alone ensures that the presupposition is supported, no constraints are placed on the global contexts, as the presupposition is guaranteed be felicitous in any global context.

As the contrast between (4-a) and (4-b) shows, not all 'other parts of the same complex sentence' seem to count equally in terms of contributing to the local context for a given presupposition. Indeed, settling which other parts of complex sentence can do this in various embedding environments is the core challenge in coming up with a precise and empirically adequate definition of the notion of local contexts. The sketch of an account of presupposition projection from conjunction, originally proposed by Stalnaker, crucially depends on the idea that the time-course of information becoming available has a crucial role to play, and that, correspondingly, linear order has a key role to play: as parts of a sentence sentence gets parsed bit by bit, information becomes available to a listener and can be added to the common ground (where appropriate). As this information becomes available from left-to-right, the resulting notion of local contexts is inherently an asymmetric one, at least for conjunction: earlier conjuncts form part of the local context for later conjuncts, but not the other way around. Correspondingly, presupposition filtering in conjunction is asymmetric, in that left-to-right filtering of presuppositions is possible, whereas right-to-left filtering is not. One key question is to what extent these properties extend to other connectives more generally.

2.1.2. SCHLENKER's LOCAL CONTEXTS. The more general question of what counts as a local context in various embedding environments comes with a key architectural choice point for theories of presupposition projection: does each connective determine what counts as local contexts for its parts individually (e.g. 'the presupposition of the second conjunct in a conjunction is evaluated in a context that contains the information of the first conjunct') or are local contexts specified in a more general way across the board? Schlenker (2009) proposes a theory along the latter lines, whose core ideas we now turn to. ${ }^{2}$

At the core of Schlenker's proposal is the idea that in determining what counts as a local context, there's an underlying strategy of only evaluating presuppositions relative to those possible worlds in which the truth value of the complex sentence overall is not already determined by other parts of the sentence. How precisely this plays out will, of course, depend on the truth-functional properties of the connective in question, which ultimately accounts for differences in local contexts, e.g., with conjunction involving consideration of information of another conjunct, whereas disjunction requires consideration of the negation of another disjunct.

To start out simple, let us again consider the conjunction in (4-b). We know that a conjunction $p \wedge q$ is true iff both $\mathrm{p}$ and $\mathrm{q}$ are true. In (4-b), $\mathrm{p}=$ 'John had prior research interests in

\footnotetext{
${ }^{2}$ The presentation here is kept fairly informal for reasons of space; see Schlenker's paper for full details.
} 
Tolkien' and $\mathrm{q}=$ 'John continues having research interests in Tolkien.' We parse $\mathrm{p}$ and subsequently 'and', thus knowing that we are dealing with a conjunction. We now need to calculate the local context against which we will evaluate the second conjunct q. Schlenker's algorithm predicts that the local context will consist of those possible worlds in which the truth or falsity of q will make a difference for the overall truth or falsity of the whole conjunction. In worlds where $\mathrm{p}$ is false, the whole conjunction as a whole will necessarily be false, and the truth or falsity of q makes no difference - $q$ can only make a difference in worlds where $p$ is true. Therefore, worlds in which $\mathrm{p}$ is false need not be considered, and the local context against which $q$ is evaluated consists only of the worlds in which $p$ is true. In the case of (4-b), these will be the worlds where 'John had prior research interests in Tolkien.' Therefore, the local context entails that John had prior research interests in Tolkien, and the presupposition to that effect introduced by continue in q, evaluated relative to this local context, does not project.

On the other hand, the presupposition trigger in (4-a) occurs in the first conjunct. In this case we cannot constrain the set of worlds based on what will end up mattering for the truth of the entire conjunction in a parallel way, thus all all worlds in the global context will need to be considered. Depending on the context, this could include worlds where John had prior research interests in Tolkien as well as worlds where he did not. Therefore, the local context will contain both kinds of worlds (assuming no support for the presupposition has been part of the prior discourse), and hence does not entail the presupposition that John had prior research interests in Tolkien. Thus, the presupposition projects.

Let us now turn to consider Schlenker's local context for (the second disjunct of) disjunctions. Take a disjunction of the form $p \vee q$. From left-to-right, $\mathbf{p}$ gets parsed, and then 'or'. A disjunction is true iff at least one of the disjuncts is true. Therefore, if $p$ is true, then the entire disjunction is bound to be true, regardless of how the second disjunct turns out. The only case where the second disjunct winds up mattering for the overall truth value then is in cases where $\mathrm{p}$ is false. Thus, the local context in which $\mathrm{q}$ is evaluated is the set of worlds where $\mathrm{p}$ is false. This predicts that a presupposition in $\mathrm{q}$ will be filtered iff it is entailed by the negation of $\mathrm{p}$. This correctly captures standardly observed projection behavior. Consider (6):

(6) Either John has never had research interests in Tolkien or he continues having research interests in Tolkien.

Intuitively, no infelicity due to the presupposition of continue arises here, just as predicted. If we extend the asymmetric perspective laid out above for conjunction to disjunction, a further prediction is that a presupposition in the first disjunct should always project, since at the time the first disjunct is parsed, nothing other than the global context precedes it, and thus the presupposition requires support in the global context:

(7) Either John continues having research interests in Tolkien or he has never had research interests in Tolkien.

This prediction does not seem to be borne out, as there doesn't seem to be any infelicity due to the presupposition of continue in this case present, despite lack of support in the global context. The question now is why this might be the case. One obvious option is to say that in the case of disjunction, filtering is symmetric, making right-to-left filtering an available option, so that the second disjunct can filter a presupposition in the first disjunct, in contrast to the asymmetric perspective on filtering spelled out for conjunction above. But as discussed in the 
following section, this is not the only possible theoretical reaction to (7).

\subsection{ACCOUNTS OF BATHROOM DISJUNCTIONS.}

2.2.1. SCHLENKER's ACCOUnT. The way that Schlenker's account deals with problems like the one that bathroom disjunctions give rise to is by taking a more nuanced view of the status of projection asymmetries. In particular, it sees them as rooted in a processing preference for left-to-right filtering. This maintains at least a certain level of asymmetry, but also opens the door for considering the underlying projection mechanisms proper to be symmetric, though with the caveat that right-to-left filtering is only available at a processing cost.

While this reconciles the phenomena observed for bathroom disjunctions with the asymmetries found for conjunctions, it fundamentally weakens the notion that filtering strongly and directly depends on left-to-right . Moreover, it raises the question of how generally symmetric filtering should be available for other connectives beyond disjunction, specifically conjunction. This becomes particularly important in light of recent results that presupposition filtering in conjunctions is asymmetric (Mandelkern et al. 2020), discussed in detail below. Since Schlenker's account aims to provide a single general mechanism for presupposition projection, - which is of course conceptually attractive - it predicts that projection asymmetries and the processing costs associated with right-to-left filtering are uniform across the board.

2.2.2. The Hirsch \& HaCkl (2014) ACCOunT. Hirsch \& Hackl (2014) pursue an alternative response to the challenge posed by bathroom disjunctions, which makes it possible to maintain a genuinely asymmetric filtering mechanism. Rather than explaining the presuppositional acceptability (7) in terms of right-to-left filtering, they suggest an alternative way of deriving the absence of a global presupposition. Since they assume that filtering does follow parsing in proceeding from left-to-right, the presupposition in the first disjunct does project, at least initially. However, the interpretation arrived at based on that winds up being discarded on the basis of more general pragmatic considerations associated with disjunctions once the second conjunct is taken into account, by using a mechanism of local accommodation.

The relevant pragmatic principle they invoke is the 'Non-Opinionatedness' constraint (NO), which states that for a disjunction ' $\mathrm{S}_{1}$ or $\mathrm{S}_{2}$ ' to be felicitous the speaker must believe that both disjuncts are live options in the discourse. Consider (8):

(8) Either Sue went to the cinema or she went to the department store.

According to NO, this disjunction is infelicitous in contexts where we know that Sue went to the cinema and did not go to the department store (or the other way around). Both disjuncts must be possible outcomes, i.e. the speaker must not think that only 'Sue went to the cinema' or only 'Sue went to the department store' is true. This follows from the maxim of quantity (Grice (1975)): if the speaker knows that only 'Sue went to the cinema' is true, then they should just assert that, similarly for 'Sue went to the department store'. Let us now consider the impact of NO on bathroom disjunctions:

(9) Either John continues having research interests in Tolkien or he has never had research interests in Tolkien before.

As the sentence is incrementally parsed, the presupposition of the first disjunct projects in an initial step, placing the standard requirement on the global context that John used to have research interests in Tolkien. However, maintaining such a global requirement would amount to 
committing to the second disjunct being false in the context (as it explicitly denies that John used to have research interests ), thus violating NO. As soon as this violation is detected, the hearer attempts to remedy this violation, and resorts to an operation of local accommodation, which provides an alternative means for preventing the presupposition from projecting.

A few words need to be said about the notion of accommodation just invoked: Accommodation is a general context-updating mechanism that hearers utilize in order to silently adjust the context when they realize that their common ground and that of their interlocutor diverge (Heim (1983)). It comes in two varieties: global accommodation, where information is added to the global common ground, and local accommodation. The focus for our purposes is the latter type, which is invoked in cases where a presupposition cannot be added to the global context for one reason or another, e.g., because that would lead to an inconsistency. To illustrate:

There is no King of France. Therefore, the King of France is not bald.

The sentence in (10) does not seem to presuppose that there is a king of France, nor does it suffer from presupposition failure of any sort. The absence of the presupposition that 'there is a king of France' cannot be due to global accommodation, given that there is no corresponding global inference. However, local accommodation has the effect of adding the information introduced as a presupposition to the local context, meaning that it will behave just like asserted content in terms of being affected by embedding operators. Thus, the presupposition will not end up affecting the global context directly, i.e., not project. While there are different specific implementations of the particular mechanism (Heim 1983; Beaver \& Krahmer 2001), this level of detail suffices for our purposes. By providing a way to avoid projection, local accommodation comes to the rescue in bathroom disjunctions as it helps to avoid the clash with NO that would arise if the presupposition were accommodated globally; effectively, it results an interpretation that can be paraphrased as follows:

(11) Either John used to have research interests in Tolkien and continues having research interests in Tolkien, or he has never had research interests in Tolkien.

Importantly, local accommodation is commonly taken to be a dispreferred option, and is accordingly assumed to be associated with a processing cost by Hirsch \& Hackl (first experimental data supporting this assumption was presented in Chemla \& Bott 2013; Romoli \& Schwarz 2015). Accordingly, their account of bathroom sentences also invokes asymmetry based on disjunct order in bathroom sentences: theoretically, only the variant allowing left-to-right filtering actually involves filtering, whereas the reverse disjunct order involves local accommodation to avoid the clash with NO. Furthermore, under the assumption that local accommodation comes at a processing cost, the version with the trigger in the first disjunct is assumed to come with a cost, in a way similar to the cost posited for symmetric filtering by Schlenker. ${ }^{3}$

2.3. Experimental BaCKGROUnd: ASymmetry in CONJUnCtion. In designing an experimental paradigm to tease apart the predictions of symmetric vs. asymmetric accounts of projection from disjunction, we adapt the methodological approach of Experiment 3 in Mandelkern et al. (2020), who investigate (a-)symmetry in conjunction experimentally. They use

\footnotetext{
${ }^{3}$ Note that Hirsch \& Hackl (2014) report experimental data from binary preference tasks that indeed suggest that bathroom disjunctions with the trigger in the second disjunct are preferred. We can't review these in detail for reasons of space, but will briefly come back to assessing their conclusions of this data in relation to our own experiments.
} 
an acceptability task, where participants were shown a context and a sentence and were asked to evaluate how natural the sentence sounds in the given context on a 7-point scale. The point of the Mandelkern et al. experiment was to investigate whether or not right-to-left filtering is available in conjunctions (as is arguably predicted by a uniform projection mechanism that is asymmetric by default, but symmetric underlyingly, such as Schlenker's). To that end they had the following conditions, illustrated with the emotive factive trigger happy (which presupposes its complement clause to be true):

a. $\quad$ COND-Ps $^{4}$ (A simple presuppositional sentence)

If Emily is happy that Jacob is in France, then she will call him soon.

b. Ps-First (A conjunction with a presuppositional first conjunct and a second conjunct that entailed the presupposition of the first conjunct)

If Emily is happy that Jacob is in France and he is in Paris, then she will call him soon.

c. Ps-SECONd (A conjunction with a presuppositional second conjunct, and a first conjunct that entailed the preuspposition of the first conjunct)

If Jacob is in Paris and Emily is happy that he is in France, then she will call him soon.

d. No-Ps-First (A conjunction like the one in Ps First, but with no presupposition in the first conjunct)

If Emily was hoping that Jacob is in France and he is in Paris, then she will call him soon.

e. No-Ps-SECOND (A conjunction like the one in Ps Second, but with no presupposition in the second conjunct)

If Jacob is in Paris and Emily was hoping that he is in France, then she will call him soon.

Two things to note: (1) the conjunctions containing the presupposition trigger are embedded in the antecedent of a conditional. This embedding is necessary in the case of conjunction in order to tease apart acceptability due to global accommodation from acceptability due to rightto-left filtering. Presuppositions project from the antecedent of conditionals, and a presupposition that is globally accommodated would thus project; in contrast, if the presupposition were filtered by the following conjunct, it should not have any impact on the global context. (2) the presupposition-bearing conjunct asymmetrically entails the presupposition-less conjunct. ${ }^{5}$

These conditions were embedded in two different types of contexts: an explicit ignorance context (EI) (which explicitly asserted that the presupposed proposition was consistent with the context but not entailed by it), and a support context (S) (which explicitly supported the presupposition). The logic is the following: an explicit ignorance context should give rise to infelicity in combination with any target sentence where the presupposition projects, as in COND-PS, because the speaker first explicitly states that they do not know whether $\mathrm{p}$, and then

${ }^{4}$ This is called SIMPLE-PS in Mandelkern et al. (2020), but we adjusted it to match our own condition names below.

${ }^{5}$ As we depart from this in our experiment due to the properties of disjunction, we do not dwell on this feature here. Its motivation stems from the need to control for any potential redundancy-induced infelicities, as 'Mary is happy that Jacob is in France and Jacob is in France' could be infelicitous not because of anything related to projection, but because the second disjunct simply reiterates information that was already added to the common ground via accommodation of the presupposition of the first conjunct. Having the asymmetric entailment avoids this confound. 
go on to presuppose that $\mathrm{p}$ in the following sentence. Whether or not infelicity arises in the PS-FIRST condition depends on whether right-to-left filtering is available: if it is, then there should be no clash with the explicit ignorance context, as the presupposition would not project; if it is not, then the discourse faces the same predicament as COND-PS.

The other conditions provide various controls. The level of acceptability of the COND-PS sentences in the explicit ignorance context gives a baseline for local accommodation, which provides a potential rescue to make this sentence acceptable in the context. Any unnaturalness of the PS-FIRST sentences that goes beyond the baseline of the COND-PS sentences can thus be attributed to projection. PS-SECOND provides a baseline of the acceptability of the overall conjunction in a case where no projection is predicted to take place (due to universally assumed left-to-right filtering). The NO-PS-FIRST/SECOND conditions provide us with a control for any effects independent of presupposition or presupposition projection (e.g. other order effects of these types of conjuncts) that might be affecting the acceptability of these conjunctions. Finally, the support context provides a control across conditions, providing a baseline point of comparison for the acceptability of the target sentences in the absence of presupposition-related infelicities.

The results of Mandelkern et al. (2020) strongly support an asymmetric view of conjunction. As can be seen in Figure 1, a PS-FIRST sentence is judged much worse in an EI context, compared to the PS-SECOND condition in the same context; moreover, it is worse in a way that goes over and above the difference between No-PS-FIRST and No-PS-SECOND. This suggests that what drives the decrease in acceptability of PS-FIRST in EI contexts is something beyond any infelicities that are due to the order of the two conjuncts, namely the fact that the presupposition projects and is not filtered (for full details, see Mandelkern et al. 2020).

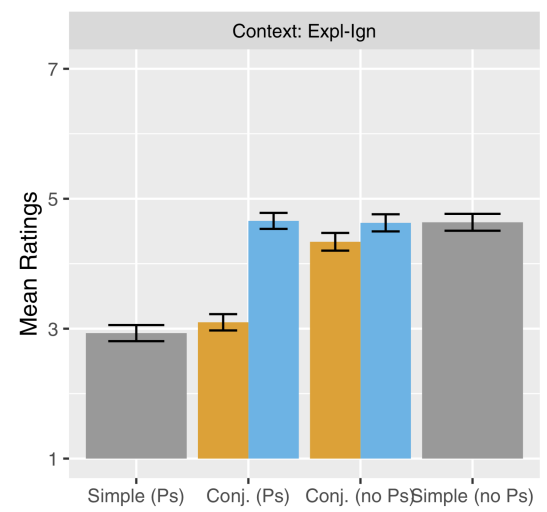

Figure 1. Mean acceptability for each condition in Mandelkern et al. (2020)

If right-to-left filtering were available for conjunction, one would expect that it would be put to use in EI contexts in order to avoid the infelicity that results from projection, even if it came at a cost, as it is the only way to rescue the discourse from being unacceptable. However, the acceptability of the PS-FIRST sentences in EI contexts is just as low as that of the COND-PS sentences. Recall that the only mechanism that allows a COND-Ps sentence to be acceptable in an EI context is local accommodation. Thus, the fact that the acceptability of PS-FIRST sentences parallels that of COND-PS sentences in EI contexts is evidence that no filtering is available in PS-FIRST sentences, and that the extent to which they are acceptable is entirely attributable to the availability of local accommodation. 
In sum, Mandelkern et al. (2020) present a strong case for filtering in conjunction to be asymmetric. In light of the success of this paradigm for testing projection (a-)symmetries in conjunction, , we adapt this approach in order to answer the corresponding question for disjunction: Do disjunctions allow right-to-left filtering of presuppositions?

\section{Experiment on Projection in Disjunction.}

3.1. DeSign. While our design adapts the general approach of Mandelkern et al. (2020), we also diverged in some important details, largely due to implementation challenges specific to looking at disjunction. We present examples of our stimuli first, and then comment on the motivations for the various differences. We created 6 Items using different triggers (continue, again, aware, find out, happy, stop), with variations in 6 conditions (in the examples below, the presupposition-bearing disjunct is underlined for presentational purposes only).

Conditionals with the trigger in the antecedent (COND-PS), (14), in Explicit Ignorance (EI) and Support (S) contexts established a baseline for the acceptability of local accommodation and a baseline for presuppositional support respectively (as in Mandelkern et al.).

(13) Contexts: My friend John researches 20th century literature. One day, I stopped by his house and I saw a copy of Tolkien's "The Fellowship of the Ring" lying around.

a. I know that John has been researching Tolkien recently, ...

b. I don't know if John has ever had research interests in Tolkien's work,... ... so I thought:

(14) If John continues having research interests in Tolkien, then that's why he is reading 'The Fellowship'.

(COND-PS)

Our disjunction variants of the PS-FIRST vs. PS-SECOND conditions are instances of 'bathroom disjunctions', as illustrated in (15)-(16): If any filtering asymmetries are present in disjunction, they should show up as differences in the acceptability between these two conditions (we turn to detailed discussion of predictions of the various accounts in the following section):

(15) Either John continues having research interests in Tolkien, or he has never had an interest in Tolkien and the book is unrelated to his research.

(PS-FIRST)

(16) Either John has never had an interest in Tolkien and the the book is unrelated to his research, or he continues having research interests in Tolkien.

(PS-SECOND)

Note that in order to increase overall discourse coherence and felicity, our non-presuppositional disjunct was expanded to include a conjunction (e.g., and the book is unrelated to his research), which intuitively helped in situating the possibility presented in that disjunct.

Finally, we included non-presuppositional disjunction control variants (No-Ps), (17)-(18). The idea is that if there is any decreased acceptability associated simply with the order in which the disjuncts are presented (in ways orthogonal to presupposition projection), then this will be reflected in the acceptability of the No-PS variants. Subsequent comparison between the PS and No-PS variants will reveal any projection-induced decrease in acceptability unique to the PS variants, which can then be attributed to presupposition-related properties.

(17) Either John has research interests in Tolkien, or he has never had an interest in Tolkien and the book is unrelated to his research.

(No-Ps-First) 
(18) Either John has never had an interest in Tolkien and the the book is unrelated to his research, or he has research interests in Tolkien.

(NO-PS-SECOND)

While our design parallels that of Mandelkern et al. (2020) closely, there are four differences:

Our target disjunction in the (NO-)PS-FIRST/SECOND conditions were not embedded in the antecedent of a conditional, as sentences following that pattern quickly get excessively complex and hard to evaluate. But conceptually, the motivation for embedding conjunctions in conditionals also doesn't extend to disjunctions, thus making this complication unnecessary: as noted above, for an unembedded conjunction, you cannot easily differentiate whether a presupposition introduced in the first conjunct might be acceptable because it can be globally accommodated, or because it has been filtered, because the information will enter the updated context either way. The same does not hold for disjunction, due to the different truth conditions, as a filtered presupposition does NOT become part of the updated context (e.g., in (6), this leaves open whether or not John has had prior research interests in Tolkien).

Even with the simplified stimuli without embedding in a conditional, bathroom disjunctions are a very particular type of sentence, and it is not easy to construct sentences and contexts that are readily comprehensible and reasonably acceptable. More importantly, participants would likely pick up even more quickly than in other cases if they keep seeing many repeated variants of the same bathroom sentence pattern, and might adopt task-specific strategies in assessing these types of items upon repeated exposure. For that reason, we opted to have a smaller set of stimuli, and to make up for the corresponding loss in statistical power by having a greater number of participants instead.

In our design, the non-presuppositional disjunct does not asymmetrically entail the presupposition introduced in the other, in contrast to Mandelkern et al. (2020) (see fn. 5). This is an unavoidable consequence of that fact that we are dealing with bathroom disjunctions, where one disjunct directly negates other. But for the same reason, the redundancy concerns motivating this feature for conjunctions do not extend to disjunction

We only presented the COND-PS condition in the SUPPORT context, as it is infelicitous to assert a bathroom disjunction in a context that explicitly supports the presupposition:

(19) (Uttered in a context where we know that the house has a bathroom)

\# Either the bathroom is in a weird place or this house has no bathroom!

This infelicity is attributable to the Non-Opinionatedness constraint posited by Hirsch \& Hackl (2014). The disjunct that expresses the non-existence of the bathroom cannot be a live option when we know that there is a bathroom. Not too much is lost by this move, however, as the sole role of the SUPPORT context is to provide a baseline for what happens when no clashes due to presupposition projection arise: in the SUPPORT context, this is achieved by having presuppositions be entailed by the global context. But the NO-PS-FIRST and No-PS-SECOND effectively serve the same general purpose, as they do not introduce any presupposition in the disjunction at all, and as a consequence, these items themselves also do not gain anything from being presented in a Support context. The final difference between our design and that of Mandelkern et al. (2020) is that we did not include filler items. This decision was based on our choice to just present 6 items to participants, all in different conditions. Since fillers generally serve to distract from experimental items, we concluded that they were not necessary, and that on balance it'd be best to keep the length of the experiment as a whole minimal. 
3.2. Predictions. Recall the main competing accounts of the projection phenomena observed for disjunction, specifically in bathroom sentences: First, projection from disjunction might be entirely symmetric (cf. conjunction), without any associated costs. Alternatively, we reviewed two accounts that do posit some level of asymmetry at one level or another:

(20) Schlenker (2009): Symmetric filtering is possible in a 'bathroom disjunction', but associated with a processing cost, due to a processing preference for asymmetric projection.

(21) Hirsch \& Hackl (2014): Presuppositions in the first disjunct of a "bathroom disjunction' DO project (maintaining that projection from disjunction is strictly asymmetric), but subsequently get locally accommodated to avoid a clash with NO; local accommodation is assumed to come with its own processing cost.

Both accounts thus posit an asymmetry of one sort or another between PS-FIRST and PsSECOND, which is associated with a processing cost, that is standardly going to be assumed to be reflected in decreased acceptability in an acceptability judgment task: Under the Schlenker (2009) view this comes about because right-to-left filtering incurs a cost, whereas for Hirsch \& Hackl (2014) this is due to the fact that local accommodation is required in Ps-FIRST sentences but not in PS-SECOND sentences, which also incurs a processing cost. Note that both Schlenker's symmetric filtering cost, and the Hirsch \& Hackl local accommodation cost are presupposition-specific and therefore should play no role in the NO-PS-FIRST/SECOND conditions. Therefore, both accounts predict that PS-FIRST and PS-SECOND should differ in acceptability to a greater extent than No-PS-FIRST and NO-PS-SECOND. In other words, both views predict an interaction between PS-FIRST/SECOND and NO-PS-FIRST/SECOND.

There is furthermore a prediction specific to the local accommodation view. The PS-FIRST and COND-PS sentences in EI contexts are parallel on the local accommodation view, in that they are both acceptable precisely to the extent that local accommodation is available. So, at least on this dimension, they should be equally acceptable (there could be other differences in acceptability reflecting, e.g., their difference in complexity). At the same time, the PS-SECOND and COND-Ps sentences in SUPPORT contexts are parallel in that in both cases, preceding material (either in the local context, in the case of PS-SECOND, or in the global context, in the case of COND-PS sentences in SUPPORT contexts) ensures that the presupposition is entailed in the respective local contexts; so, both the COND-PS sentences in SUPPORT contexts and the PS-SECOND sentences should be fully acceptable with regards to evaluating the presupposition. Taking these two parallels together, this means that the local accommodation account predicts that there should be no interaction between the conditions posited to involve local accommodation (Cond-Ps in Explicit Ignorance Context and Ps-First), and the conditions where the presupposition is supported in its local context (COND-Ps in Support/ Ps-SECOND). Our statistical analysis will correspondingly focus on assessing these predictions.

3.3. PARTICIPANTS \& PROCEDURE. 255 participants were recruited via Prolific, and after seeing informed consent, each was shown 6 items, one per trigger and condition, in a Latin square design. The COND-PS controls were shown first to establish baselines (either in an EI or S context, in random order), followed by the disjunction conditions (in random order). Participants indicated on a 7-point scale how natural the sentence sounds in the given context. A demonstration version as well as the underlying code and the csv-file containing the full stimuli are accessible at https://farm.pcibex.net/r/bMqAbG/. Click on 'Click here to edit a copy in the PCIbex Farm.' 
in the top bar to access code and stimuli directly (no account or sign-in needed) on the PCIbex Farm (Zehr \& Schwarz 2018).

3.4. RESUlTS. The overall pattern is simple (Fig. 2), and confirmed by mixed effect model analyses. The results are summarized in Table 1: S-PS-COND was rated higher than all other conditions, and there are no contrasts in the disjunction conditions. To confirm the statistical significance of the theoretical contrasts of interest, we analyzed the data using mixed effect regression models. First, we constructed a model comparing the individual conditions, with subjects and items as random effects (without random slopes, as more complex models did not converge). Setting the S-PS-COND as the baseline, we found it to be significantly different from all other conditions, confirming that we are able to detect differences in acceptability due to presuppositional support, as EI-PS-COND in particular is significantly less acceptable than S-Ps-Cond, but does not significantly differ from the Ps conditions (see top section of Table 1 for details of the statistical results). The disjunctions are overall less acceptable than S-PS-COND, regardless of presence of a presupposition or its status, presumably due to the additional complexity of the disjunctive sentences. Importantly, the PS-FIRST is not significantly less acceptable than the No-PS conditions, nor than the PS-SECOND condition.

Homing in on the prediction of asymmetric accounts of an interaction between PS/No-PS and Order, we subset the data to the disjunctive conditions, and centered both factors in a $2 \times 2$ interaction analysis, but found no interaction, contrary to asymmetric predictions.

Finally, we tested the prediction of local accommodation-based asymmetry accounts of an interaction between EI-COND-PS/PS-FIRST (which both require local accommodation on this account) and S-COND-PS/PS-SECOND (both of which involve a presupposition that is satisfied in its local context), again subsetting the data to the relevant conditions and centering the predictors. The interaction was found to be significant (see the last line of Table 1 for details), contrary to the local accommodation-based accounts (there also were significant main effects of both factors, but these are clearly dominated by the interaction, given the pattern in the data and the absence of significant differences between the critical disjunction conditions).

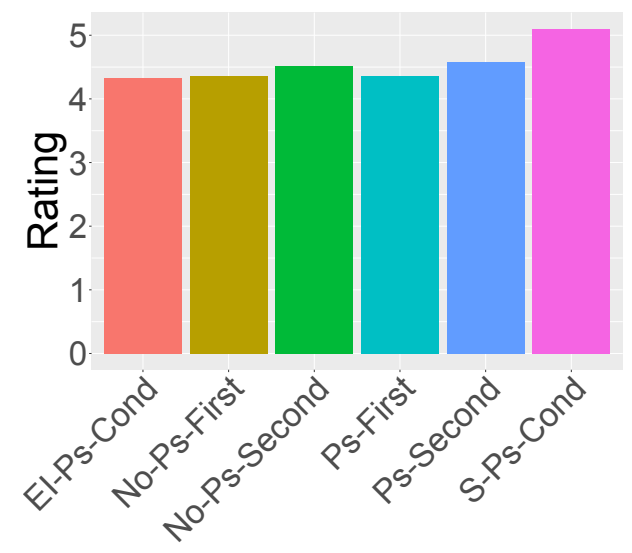

Fig. 1. Mean acceptability by condition

\begin{tabular}{l|l|l|l}
\hline S-Ps-Cond vs. & Coeff. & SE & $p$ \\
\hline \hline EI-Ps-Cond & -.89 & 0.18 & $<.001$ \\
\hline No-Ps-First & -0.78 & 0.18 & $<.001$ \\
\hline No-Ps-Second & -.54 & 0.18 & $<.01$ \\
\hline Ps-First & -0.73 & 0.18 & $<.001$ \\
\hline Ps-Second & -0.61 & 0.18 & $<.001$ \\
\hline \hline Interaction: & Coeff. & SE & $p$ \\
\hline Embed * Ps-Status & -.81 & 0.25 & $<.01$ \\
\hline
\end{tabular}

Table 1. Mixed-effect models summaries

3.5. Discussion. Both the Schlenker (2009) and the Hirsch \& Hackl (2014) views posit that something extra, beyond the default and easily available projection mechanism, is at play in PS-FIRST disjunctions (costly right-to-left filtering for the former, local accommodation for the latter). Thus, the lack of an interaction between PS-FIRST/SECOND and No-PS-FIRST/SECOND 
is unexpected under these approaches. Moreover, the prediction that was specific to the local accommodation account, i.e. that there should be no interaction between PS-FIRST/SECOND and EI-COND-PS/S-COND-PS, is also not borne out, as we do find such an interaction.

Instead, the picture that emerges from our data is one where both PS-FIRST and PS-SECOND do not exhibit any differences in acceptability. In this respect, our data represent a stark contrast to the findings for conjunction in Mandelkern et al. (2020), where their conjunctive PSFIRST was found to be significantly less acceptable than the PS-SECOND counterpart. ${ }^{6}$ Given the parallel paradigms between our experiment and that of Mandelkern et al., this difference is strong evidence that conjunction and disjunction are different in terms of their projection behavior. Thus, the symmetry that we find between PS-FIRST and PS-SECOND for disjunction suggests that the properties of presupposition projection for disjunction need to be stated independently of those for conjunction: no general mechanism that derives uniform projection properties, specifically with regards to the role of linear order, can account for these differences between 'and' and 'or'. The difference thus must come about due to differences in lexical properties relative to the mechanisms behind presupposition projection.

Generally speaking, symmetric projection behavior in disjunction is compatible with two different ways of stating the projection properties of disjunction:

Option 1 is that filtering is not at play in disjunction, and such a position has indeed been advanced, by claiming that presuppositions in general project from both disjuncts (cf. Geurts (1999)). This ensures that the two disjuncts behave symmetrically. However, more needs to be said about why neither disjunct projects in 'bathroom' disjunctions. One potential move here is to claim that local accommodation can come to the rescue in either disjunct order, and is thus responsible for preventing projection in both cases. This would give an account of why both PS-FIRST and PS-SECOND are on par in acceptability with the local accommodation conditions EI-PS-COND However, note that under such a view we might expect a contrast between PS-FIRST/SECOND and No-PS-FIRST/SECOND, as the former will involve local accommodation, whereas the latter do not. However, no such contrast is found in our data set. instead Ps-First/Second is as acceptable as No-Ps-First/Second. Without an independent point of comparison for local accommodation in disjunction (e.g., in a non-bathroom disjunction in an explicit ignorance context), we cannot conclusively assess the extent to which this is problematic.

Option 2 is that in the case of disjunction, symmetric filtering is available without incurring an extra cost (at least none that is measurable in our task). This would capture the observed symmetry between PS-FIRST and PS-SECOND, and also explain why there is no contrast between PS-FIRST/SECOND and No-PS-FIRST/SECOND. Taking this view, one might wonder why PS-FIRST/SECOND is comparable in acceptability to EI-PS-COND, even though the latter involves local accommodation. However, our results do not differentiate between the cost incurred by local accommodation (measured in EI-PS-COND) and the potential impact

\footnotetext{
${ }^{6}$ While we are not able to go into any detailed comparison with other related prior experimental work using different tasks, it's worth noting that our findings align rather well with those for disjunction in Chemla \& Schlenker (2012). At the same time, they do contrast somewhat with those in the experiments reported by Hirsch \& Hackl (2014), as their task requiring a forced choice between the two disjunct orders in bathroom sentences does indicate some level of asymmetry. However, this need not directly contradict our interpretation of the findings presented here. First, their asymmetry could directly result from the particular task, which requires explicit comparison between the two variants. Secondly, our findings are not in principle incompatible with some amount of processing advantages of left-to-right processing, which our task may not pick up on.
} 
on acceptability of the increased complexity involved in disjunction (measured in No-PSFIRST/SECOND), so the results as they stand do not pose any particular problem in this regard.

Based on the current results, we cannot decide between Option 1 and Option 2. Nevertheless, it is important to note that both Option 1 and Option 2 are incompatible with a domain general account of the projection mechanism that is common across connectives and gives rise to uniform processing costs based on preferences for left-to-right filtering: disjunction behaves symmetrically, while conjunction behaves asymmetrically; such a difference requires capturing the projection behavior for each connective separately. This means that some part of the lexical entry of the two connectives differs, either directly, by hard-wiring projection properties concerning linear order into the lexical entry, or indirectly, by identifying distinct lexical properties of the connectives (most plausibly their truth-conditions) that could interact in the appropriate way with a general projection mechanism.

4. Conclusion. We have presented experimental evidence that presupposition projection from disjsunction is symmetric. This contrasts starkly with the result in Mandelkern et al. (2020) that conjunction behaves asymmetrically. We argue that this means that a domain-general account of projection that predicts uniform left-to-right asymmetries across connectives cannot account for this contrast. Instead, we propose that the projection behavior of connectives needs to be tied to distinct properties at the lexical level.

\section{References}

Beaver, David \& Emiel Krahmer. 2001. A partial account of presupposition projection. Journal of Logic, Language and Information 10. 147. https://doi.org/10.1023/A:1008371413822.

Chemla, Emmanuel \& Lewis Bott. 2013. Processing presuppositions: Dynamic semantics vs pragmatic enrichment. Language and Cognitive Processes 38(3). 241-260. https://doi.org/10.1080/01690965.2011.615221.

Chemla, Emmanuel \& Philippe Schlenker. 2012. Incremental vs. symmetric accounts of presupposition projection: an experimental approach. Natural Language Semantics 20. 177226. https://doi.org/10.1007/s11050-012-9080-7.

Geurts, Bart. 1999. Presupposition and pronouns. Oxford: Elsevier.

Grice, Herbert P. 1975. Logic and conversation. In Peter Cole \& Jerry Morgan (eds.), Syntax and semantics III: Speech acts, 43-58. New York: Academic Press.

Heim, Irene. 1983. On the projection problem for presuppositions. In Michael Barlow, Daniel P. Flickinger \& Nancy Wiegand (eds.), Proceedings of WCCFL 2, 114-125. Stanford: CSLI.

Hirsch, Aaron \& Martin Hackl. 2014. Incremental presupposition evaluation in disjunction. Proceedings of NELS 44. 177-190.

Karttunen, Lauri. 1973. Presuppositions of compound sentences. Linguistic inquiry 4(2). 169193. http://www.jstor.org/stable/4177763.

Mandelkern, Matthew, Jérémy Zehr, Jacopo Romoli \& Florian Schwarz. 2020. We've discovered that projection across conjunction is asymmetric (and it is!). Linguistics and Philosophy 43(5). 473-514. https://doi.org/10.1007/s10988-019-09276-5.

Romoli, Jacopo \& Florian Schwarz. 2015. An experimental comparison between presuppositions and indirect scalar implicatures. In Florian Schwarz (ed.), Experimental perspectives on presuppositions, 215-240. Cham: Springer International Publishing. 
Schlenker, Philippe. 2009. Local contexts. Semantics and Pragmatics 2(3). 1-78. https://doi.org/10.3765/sp.2.3.

Stalnaker, Robert. 1974. Pragmatic presuppositions. In Milton K. Munitz \& Peter K. Unger (eds.), Semantics and philosophy, 197-213. New York University Press.

Zehr, Jérémy \& Florian Schwarz. 2018. PennController for Internet Based Experiments (IBEX). https://doi.org/10.17605/OSF.IO/MD832. 